\title{
¿Arqueología salvadoreña? Una utopía en construcción
}

\author{
Salvadorean Archaeology? A utopia in the making
}

\author{
Marlon Escamilla \\ Coordinador de la Licenciatura en Arqueología de la Utec \\ marlon.escamilla@utec.edu.sv
}

Recibido: 17/03/2015 - Aceptado: 25/03/2015

\section{Resumen}

Al hablar de arqueología en El Salvador, es necesario generar una discusión en torno a diferentes aspectos socioculturales y políticos que han influenciado la práctica de la disciplina arqueológica en el país. Desde sus inicios, la arqueología en El Salvador ha sido influenciada principalmente por los planteamientos metodológicos y teóricos de la arqueología norteamericana. A mediados de la década de los noventa se funda la carrera de Arqueología en El Salvador, permitiendo formar arqueólogos nacionales. Sin embargo, a pesar de las dos décadas transcurridas y de los más de treinta arqueólogos graduados con que cuenta el país, todavía no se puede hablar de una arqueología salvadoreña ¿Por qué? ¿Cuáles son las razones que impiden que la arqueología salvadoreña crezca y se desarrolle como tal? El presente artículo analiza y discute algunos aspectos que están afectando y atrasando el crecimiento y la madurez de la arqueología como disciplina.

\section{Palabras clave}

Arqueología nacional, arqueología salvadoreña, patrimonio arqueológico, El Salvador.

\begin{abstract}
To talk about archaeology in El Salvador, it is necessary to generate a discussion on different political and socio-cultural aspects that influenced its practice in the country. Since its inception, archaeology in El Salvador has been mainly influenced by American methodological and theoretical approaches. The Archaeology Major in El Salvador was first offered in the mid-nineties, thus allowing the training of national archaeologists. However, in spite of these two decades and the more than thirty graduates in the country, we are still unable to talk about a Salvadoran archaeology. Why? What are the reasons that prevent Salvadoran archaeology from growing and developing as such? This paper analyzes and discusses some issues that are affecting and slowing the growth and maturity of this field as a discipline.
\end{abstract}

\section{Keywords}

National archaeology, Salvadoran archaeology, archaeological heritage, El Salvador.

\footnotetext{
1 Marlon V. Escamilla, es coordinador de la Licenciatura en Arqueología de la Universidad Tecnológica de El Salvador. arqueólogo. Es candidato a Doctor en Antropología de la Universidad de Vanderbilt, Nashville, Tennessee. Por más de una década formó parte del equipo de investigadores del Departamento de Arqueología de El Salvador, fundando los Proyectos Nacionales de Arqueología Subacuática (2005) y de Arte Rupestre de El Salvador (2006). Ha publicado diversos artículos académicos relacionados con la arqueología y participado como conferencista en congresos y simposios en países como El Salvador, Guatemala, Nicaragua, Panamá, México, Israel, Colombia, Jamaica, Perú y Estados Unidos. Correo electrónico: marlon.escamilla@utec.edu.sv.
} 


\section{Introducción}

Los reconocimientos etnográficos y las descripciones de algunos sitios arqueológicos realizados por el diplomático estadounidense Ephraim George Squier, durante 1853, en El Salvador pueden considerarse como el inicio de la arqueología en El Salvador. Posterior a las descripciones de Squier, diversos exploradores y eruditos, tanto extranjeros como nacionales, brindaron destacados aportes a la arqueología en el país, dentro de los cuales se destacan el viajero alemán Simeón Habel, el historiador salvadoreño Dr. Santiago Barberena, el filólogo alemán Walter Lehman, Atilio Peccorini, Herbert Spinden, el profesor salvadoreño Jorge Lardé, el arqueólogo norteamericano Samuel Lothrop, el salvadoreño Antonio Sol, los arqueólogos norteamericanos John Dimick y Maurice Ries, el arqueólogo norteamericano John Longyear (Longyear, 1944) y el arqueólogo Stanley Boggs (Boggs, 1944), de origen norteamericano, considerado como uno de los pioneros de la arqueología salvadoreña y como uno de los arqueólogos más influyentes de la época, dirigiendo importantes proyectos arqueológicos, enfocándose en el occidente del país, en el departamento de Santa Ana, el área de Chalchuapa, específicamente en el sitio arqueológico Tazumal durante las temporadas 1943-45, 1948-50 y 1951-53; y en el departamento de San Salvador durante el año 1954 entre otros. Todos los investigadores anteriores de una u otra manera contribuyeron a cimentar las bases de la naciente disciplina de la arqueología en El Salvador.

La segunda mitad del siglo XX estuvo marcada por la experimentación de diversas metodologías y técnicas de investigación, así como marcó el inicio de nuevos intereses teóricos en la práctica de la arqueología, destacándose los trabajos de Wolfgang Haberland (Haberland, 1958) en la Gruta del Espíritu Santo, Corinto; Robert Sharer (Sharer, 1978), en Chalchuapa; Willys Andrews (Andrews, 1986), en el sitio de Quelepa; William Fowler y Howard Earnest (Fowler, William y Howard Earnest, 1985), con el Proyecto Cerrón Grande; Payson Sheets (Sheets, 2006), con el Proyecto Protoclásico en el Valle de Zapotitán; Karen Brunhns (Bruhns K. , Cihuatan: An early Postclassic town of El Salvador: The 1977-1978 Excavations, 1980) y William Fowler (Fowler, The Pipil-Nicarao of Central America., 1981) (Fowler, The Cultural Evolution of Ancient Nahua Civilizations: the Pipil-Nicarao of Central America, 1989), en el sitio arqueológico de Cihuatán; Arthur Demarest (Demarest, 1986), en el sitio Santa Leticia ubicado en la Cordillera Apaneca-Ilamatepec.
En el transcurso la década de 1980, las condiciones sociopolíticas de El Salvador no permitieron el desarrollo de investigaciones arqueológicas de una manera intensa y permanente. Lo anterior se debió al estallido del conflicto armado. No obstante, a pesar de la inestabilidad política del país, algunas investigaciones puntuales fueron desarrolladas. Blas Román Castellón (Castellón, 1992) publica un cuadro del número de publicaciones sobre la arqueología de El Salvador por década desde 1850 hasta 1990, en el cual destaca la década de 1980 como la que contó con mas publicaciones. Probablemente lo anterior esté asociado a la productiva década de 1970, en la cual se desarrollaron proyectos de investigación arqueológica de gran magnitud. Dentro de las investigaciones desarrolladas en las décadas de 1980 y 1990 destacan las siguientes: el proyecto de rescate del embalse de la represa San Lorenzo; Paul Amaroli (Amaroli, Cara Sucia: nueva luz sobre el pasado de la Costa Occidental de El Salvador, 1984), junto con Manuel Murcia y Jorge Mejía, desarrolla un proyecto arqueológico en Cara Sucia: el Proyecto Cuscatlán, dirigido por Amaroli. Arthur Demarest junto con Paul Amaroli, Bárbara Arroyo y Mary Pye desarrollan excavaciones en el sitio El Carmen en la costa de Ahuachapán; el Proyecto en el valle del río Ceniza dirigido por Kathryn Sampeck y Howard Earnest; el Proyecto Izalco, dirigido por William Fowler; el Proyecto Joya de Cerén, dirigido por Payson Sheets (Sheets, 2006); el Proyecto Arqueológico Ciudad Vieja, dirigido por William Fowler (Fowler Jr., William R., Francisco Estrada-Belli, Jennifer R. Bales, Matthew D. Reynolds y Kenneth L. Kvamme, 2007) y el Proyecto de Investigaciones Interdisciplinarias desarrollado en Chalchuapa, específicamente en el sitio Casa Blanca, el cual fue dirigido por Kuniaki Ohi (Ohi, 2000). Cabe aclarar que no se han mencionado todos los proyectos desarrollados, sino solamente algunos de ellos, considerados los más destacados.

\section{5: Inicio de la Licenciatura en Arqueología en El Salvador}

Durante el año 1994, un grupo de personas visionarias fundan la Universidad San Jorge en El Salvador. Para el año de 1995 la universidad abre sus puertas con una oferta académica de tres licenciaturas: en Arqueología, en Antropología y en Historia. Lamentablemente, la visión del grupo fundador de la Universidad San Jorge es interrumpida en el año 1997, debido a la reforma de educación superior que se implanta a escala nacional. En base a lo anterior, la universidad es 
obligada a cerrar, principalmente por falta de activos. A raíz de dicha situación, la Universidad Tecnológica de El Salvador (Utec), con una actitud de compromiso por desarrollar las denominadas carreras de la cultura, decide absorber a la Universidad San Jorge con el objetivo de continuar con la formación de profesionales en las ramas anteriormente mencionadas. Para el año 2000, la primera promoción de arqueólogos nacionales formados en el país se gradúa, y fue conformada por cuatro arqueólogos y una arqueóloga; y con temas de tesis diversos. Con esto, la arqueología salvadoreña inicia una nueva etapa. Por primera vez en la historia nacional, arqueólogos salvadoreños formados en el país ejercen la disciplina. La formación académica estuvo a cargo de profesionales nacionales y extranjeros, destacándose profesionales japoneses y estadounidenses. Actualmente se han graduado más de una treintena de arqueólogos y arqueólogas.

La segunda mitad de la década de los noventa constituye un momento histórico para la arqueología de El Salvador, debido a que por primera vez una universidad salvadoreña formaba profesionales en arqueología. Así mismo, en el año 1997 los cinco estudiantes de arqueología son contratados como técnicos para trabajar a medio tiempo en el Departamento de Arqueología del extinto Consejo Nacional para la Cultura y el Arte (Concultura), bajo la dirección de un arqueólogo salvadoreño graduado en el hermano país de Guatemala. En términos generales, la década de los noventa es una época clave y determinante en el rumbo de la arqueología de El Salvador.

Desde la apertura de la Licenciatura en Arqueología en 1995 hasta la actualidad, la Utec ha graduado un total de 33 arqueólogos, dieciséis mujeres y diecinueve hombres. Los temas de tesis desarrollados por estos son diversos, destacando en su mayoría las investigaciones relacionadas con el análisis tipológico cerámico, análisis tipológico de materiales culturales líticos y en menor porcentaje temas relacionados con la arquitectura, patrones de asentamiento $\mathrm{y}$ de enterramiento $\mathrm{y}$ temas vinculados con la sensibilización y educación. Así mismo, de los 33 arqueólogos, ocho cuentan con maestrías enfocadas en las áreas de la arqueología, la antropología, la historia, las ciencias sociales, la conservación del patrimonio cultural y el desarrollo territorial. En su mayoría, los títulos de maestría han sido obtenidos en universidades extranjeras de países como Estados Unidos, Canadá, España, México, Guatemala y Japón. En la actualidad, dos arqueólogos están en categoría de doctorando, uno por una universidad estadounidense y otro por una universidad española.

Han transcurrido ya dos décadas desde la apertura de la carrera de Arqueología en El Salvador. Mucho se ha avanzado desde aquel tiempo (Paredes y Erquicia, 2013; Valdivieso, 2010; Erquicia, 2011; Escamilla y Fowler, 2011; Cobos, 1994), no solamente en la formación académica sino al abrirse espacios académicos de discusión, como el Foro de Estudiantes de la Escuela de Antropología (Foesa) de la Utec. Dicho foro se celebra desde el 2006 de manera anual. Este año se llevará a cabo el décimo Foesa. Dentro de las fortalezas de este se destaca que los organizadores son los mismos estudiantes de las licenciaturas de Arqueología y Antropología, permitiéndoles generar habilidades y competencias tanto organizativas como participativas en eventos académicos.

Otro logro importante es la organización del Congreso Centroamericano de Arqueología, el cual se inauguró en el año 2005 y se desarrolla cada dos años. Durante el mes de noviembre de este año se realizará el sexto congreso, permitiendo la concentración de académicos de diversas universidades e instituciones, tanto a escala nacional como regional. El congreso es importante porque genera discusión académica y posiciona la arqueología de El Salvador regionalmente.

Como es de esperarse, y a pesar de los avances realizados hasta la fecha, la arqueología en El Salvador aún continúa atravesando serias dificultades relacionadas con su conformación y consolidación.

\section{Invisibilización de los arqueólogos nacionales}

Los inicios de la arqueología en El Salvador, como en muchos países de la región centroamericana, han estado íntimamente ligados e influenciados por el desarrollo de proyectos arqueológicos dirigidos por extranjeros. Sin embargo, la presencia de estos en El Salvador no solo ha estado limitada a la dirección de proyectos, sino también a la dirección y asesoría tanto de departamentos como de direcciones afines de la institución gubernamental a cargo de la salvaguarda, protección y manejo del patrimonio cultural arqueológico.

Durante la década de 1960, el antiguo Departamento de Excavaciones Arqueológicas de El Salvador estaba bajo la 
jefatura del salvadoreño Alfonso Huezo Córdoba, quien, aunque no tenía un título académico que lo acreditara como arqueólogo, desarrollo diversas investigaciones visitando, documentando y registrando varios sitios arqueológicos a escala nacional. Aproximadamente a mediados de la década de 1960, la jefatura del Departamento de Excavaciones Arqueológicas fue asumida por el arqueólogo norteamericano Stanley Boggs, quien es considerado pionero de la arqueología en El Salvador. Boggs estuvo al frente de ese departamento desde 1965 hasta 1991, año en el que fallece. Sin embargo, durante un corto lapso, de 1986 a 1988, la jefatura del Departamento de Arqueología es asumida por el arqueólogo salvadoreño Gregorio BelloSuazo. Durante el período comprendido entre 1992 y 1994 , el Departamento de Arqueología estuvo bajo la dirección del arqueólogo norteamericano Paul Amaroli. Durante el año 1995, y por un corto período de meses, la dirección del Departamento de Arqueología estuvo a cargo del antropólogo suizo Emmanuel Broillet. Desde 1965 hasta 1995 - a excepción de la corta dirección de Bello-Suazo-, la arqueología de El Salvador fue dirigida por extranjeros, abarcando un lapso de casi tres décadas. Lo anterior es entendible tomando en cuenta que no existía la carrera de Arqueología en el país. Si alguien quería estudiar dicha disciplina, tenía que salir del país para poder formarse como arqueólogo.

Las anteriores tres décadas mencionadas fueron muy importantes para la arqueología de El Salvador, debido a que durante este período de la historia de la arqueología muchos investigadores extranjeros vinieron al país a desarrollar sus tesis doctorales con temáticas diferentes, lo cual fue positivo para el conocimiento arqueológico nacional. Fue una lástima solo algunos de esos investigadores continuaron desarrollando su trabajo en el país. La mayoría solamente realizó sus tesis doctorales; pero desarrolló sus carreras académicas en otros países de la región.

La permanencia de los investigadores que decidieron continuar desarrollando sus carreras académicas en el país fue importante debido a que, por un lado, muchos proyectos de investigación iniciados por ellos se han continuado desarrollando, y por otro, varios arqueólogos salvadoreños se han beneficiado con becas de maestrías y doctorados en el extranjero gracias a su apoyo, contribuyendo de esa forma al desarrollo de la arqueología en El Salvador. Así mismo, se implantaron proyectos regionales en ciertas áreas del país, contribuyendo al registro y documentación de sitios arqueológicos. Otro aporte fue la conformación de la Ley Especial para la Protección del Patrimonio Cultural y su Reglamento, la cual fue puesta en vigencia en el año de 1993.

A partir de 1995, hasta 2008, la arqueología de El Salvador dio un giro interesante debido a que el puesto de jefatura del Departamento de Arqueología estuvo a cargo de diversos arqueólogos salvadoreños, no obstante la escasa presencia de arqueólogos nacionales graduados. Desde 1995 hasta mediados de 1997, el Departamento de Arqueología estuvo a cargo del arqueólogo salvadoreño Fabio Amador, quien había sido formado en arqueología en una universidad estadounidense. De 1997 a 2000, el Departamento estuvo a cargo del arqueólogo salvadoreño José vicente Genovés, quien obtuvo su grado de Licenciatura en la Universidad San Carlos de Guatemala. Posteriormente $-\mathrm{y}$ por primera vez en la historia de El Salvador-, la jefatura del Departamento de Arqueología es asumida por un arqueólogo salvadoreño graduado en el país, Roberto Gallardo, quien se formó y graduó en la Utec. Gallardo estuvo al frente del Departamento de 2000 a 2002. Luego, de 2002 hasta principios de 2008, este estuvo bajo la responsabilidad del arqueólogo salvadoreño Fabricio Valdivieso, que también se había formado y graduado de la Utec. Infelizmente este período sería el último en que un arqueólogo salvadoreño estuviera atendiendo el cargo.

Durante este período, de unos 12 años, muchos logros se alcanzaron, dentro de los cuales se destaca la implantación de una normativa de investigaciones arqueológicas; se fundan y se desarrollan proyectos nacionales como el Atlas Arqueológico de El Salvador, Proyecto Arte Rupestre de El Salvador, Proyecto Arqueología Subacuática de El Salvador, proyectos de Arqueología Histórica; se implantaron las Sesiones Científicas y el Congreso Centroamericano de Arqueología, espacios académicos en los cuales se discute sobre temáticas del quehacer arqueológico de El Salvador. Así mismo, la mayoría de investigaciones eran socializadas en congresos y simposios, tanto a escala nacional como internacional. Desde 2008 hasta la actualidad la Dirección de Arqueología ha estado a cargo del arqueólogo japonés Shione Shibata.

Al realizar un simple análisis numérico de los arqueólogos salvadoreños graduados presentes en el país, se obtienen datos interesantes. Durante el período de doce años, comprendido entre 1995 a 2008, el número de arqueólogos 
y arqueólogas graduados era de diez, de los cuales cinco trabajaban en el Departamento de Arqueología, es decir, el $50 \%$. A pesar de la ausencia de graduados al inicio de este período, y del escaso número de graduados a partir del 2000, la jefatura del Departamento de Arqueología siempre estuvo a cargo de un arqueólogo salvadoreño. Por otro lado, en el período comprendido de 2009 hasta la actualidad, el número de graduados es de 33 , de los cuales doce laboran en diferentes puestos de la Secretaría de Cultura de la Presidencia (Secultura), es decir, el 36,36\%. A diferencia del período anterior, para el 2009 había trece arqueólogos graduados, de los cuales tres se encontraban fuera del país. Actualmente, tres arqueólogos se encuentran en proceso de elaboración de tesis. A pesar de haber aumentado el número de arqueólogos graduados al $230 \%$, este período se ha caracterizado por la ausencia de arqueólogos o arqueólogas que estén al frente de la Dirección de Arqueología. Aunque es imposible pensar que la Secultura sea la única fuente laboral para los arqueólogos. Es de reconocer que desde el año 2009 hasta el 2014 el Estado ha realizado grandes esfuerzos para la apertura de nuevas plazas.

\section{El manejo del patrimonio cultural arqueológico en El Salvador}

La Secultura tiene bajo su responsabilidad el patrimonio cultural de El Salvador. Esta institución estatal es la que vela por la conservación, salvaguarda e investigación del patrimonio cultural tangible e intangible del país. Dentro de dicha institución se encuentra la Dirección de Arqueología, a la cual le compete el manejo integral del patrimonio cultural arqueológico; y actualmente cuenta con ocho arqueólogos nacionales que desempeñan cargos de técnicos, y un arqueólogo extranjero que es el director.

Los datos anteriores permiten formular varias preguntas. ¿Por qué, a veinte años de haberse abierto la oportunidad de estudiar la carrera de arqueología en El Salvador, la Dirección de Arqueología sigue siendo dirigida por un arqueólogo extranjero? ¿Cuáles son las razones primarias de la Secultura para no permitirle a un arqueólogo, o a una arqueóloga, salvadoreño asumir esa Dirección?

La primera respuesta que se le viene a la mente de colegas de otros países es que no conocen muy bien la situación actual de la arqueología en El Salvador; es que en El Salvador no existen arqueólogos salvadoreños. Es más, muchos coterráneos piensan de la misma forma. Es totalmente penoso que en la actualidad El Salvador sea el único país de la región centroamericana que cuenta con un arqueólogo extranjero al frente de la Dirección de Arqueología. Y lo es aún más cuando la población salvadoreña en general y los colegas de otros países se dan cuenta de que sí existen 33 arqueólogos salvadoreños. $Y$ surge la pregunta obvia: ¿Entonces, por qué está un extranjero como director? Innumerables veces esta pregunta ha quedado sin respuesta debido a lo incomprensible que resulta tal situación. El mensaje que el Estado envía a los colegas al sostener y respaldar esta situación es que aparentemente de los 33 arqueólogos y arqueólogas salvadoreñas ninguno tiene la capacidad suficiente para asumir la dirección de Arqueología, lo cual es rechazable desde cualquier punto de vista.

El problema no puede ser asociado a la falta de plazas o personal dentro de la institución. De los 33 arqueólogos, 12 trabajan en el Secultura, lo cual irónicamente es una cifra récord, ya que nunca, según la historia de la arqueología de El Salvador, habían trabajado tantos arqueólogos en la institución estatal. Muchas variables afloran ante tal situación. ¿Estará asociada con el malinchismo esta situación? Lamentablemente, buena parte de nuestra historia nacional ha estado ligada a la conducta como la de la Malinche, en la cual se tiende a creer en la superioridad de lo extranjero sobre lo nacional. ¿Habrá de por medio con intereses diplomáticos? Los vínculos entre naciones tienden a estar asociados a compromisos entre ambas partes.

Aunque no hay una respuesta certera ante tal situación, lo que sí es cierto es la existencia de un proceso de invisibilización de los arqueólogos salvadoreños por parte del Estado. Recientemente se nombró como director del Museo Nacional de Antropología "Dr. David J. Guzmán" al arqueólogo salvadoreño José Heriberto Erquicia, hecho digno de resaltar y felicitar. ¿Por qué no hacer lo mismo con la Dirección de Arqueología? El cuestionamiento no está asociado con un problema de xenofobia, ni mucho menos personalizado con el actual director. Al contrario, en el proceso de formación educativa de los estudiantes de la carrera de Arqueología se cuenta con varios arqueólogos extranjeros como docentes.

El problema radica en el hecho de que el arqueólogo al frente de la Dirección de Arqueología debe desarrollar procesos de apropiación e identificación con el patrimonio cultural arqueológico, que permitan la conservación y salvaguarda 
de dicho patrimonio, en la mayor acepción del término, lo cual tiende a ser difícil cuando no se conoce la cultura de un país. Así mismo, no tiene sentido formar estudiantes salvadoreños cuando ellos mismos saben de la existencia de un proceso de invisibilización, en el cual el mayor referente de la arqueología salvadoreña es un extranjero.

\section{¿Arqueología salvadoreña?}

El manejo del patrimonio arqueológico de una nación es sumamente delicado, pues se requiere una compenetración y su identificación total, conociendo los aspectos que lo componen. A lo largo de la historia, a través de la arqueología se ha creado identidad y nacionalismo en diferentes partes del mundo. Un ejemplo emblemático es México, cómo el Estado decidió invertir en investigaciones arqueológicas hace más de un siglo con el objetivo de generar nacionalismo e identidad.

¿Existen proyectos arqueológicos nacionales permanentes con temporadas de campo anuales y con una visión de corto plazo que por lo menos abarque un período de cinco años? No. ¿Existen proyectos nacionales de sensibilización con el patrimonio arqueológico a escala nacional? No. ¿Existen proyectos arqueológicos nacionales que tengan como finalidad la conformación de identidades? Y la respuesta nuevamente es no. ¿Por qué no existen? Probablemente la respuesta está asociada, por un lado, a carecer de una visión y proyección del manejo del patrimonio cultural arqueológico salvadoreño, y por otro, a la falta de presupuesto.

Actualmente no es posible hablar de una arqueología salvadoreña como tal. Se han desarrollado, y se siguen desarrollando, grandes esfuerzos en la conformación y consolidación de la disciplina en el país. Sin embargo, son muchos los retos y desafíos que demanda la arqueología de El Salvador en la actualidad. ¿Qué se necesita cambiar de la situación actual? Una de las primeras acciones que se debe realizar es que la Dirección de Arqueología de la Secultura debe de ser delegada a un arqueólogo 0 arqueóloga salvadoreños. Es inaceptable que, a dos décadas de la apertura de la carrera de arqueología y contando con 33 arqueólogos graduados, se siga dejando el manejo del patrimonio arqueológico salvadoreño en manos extranjeras. Sin duda, cada uno de los arqueólogos graduados tiene la capacidad y competencia para desempeñar el cargo de director de Arqueología.
Así mismo, es necesario plantear y desarrollar proyectos arqueológicos nacionales permanentes, con temporadas de campo anuales que permitan una continuidad de, por lo menos, cinco años, en los cuales se involucre a estudiantes nacionales de la carrera de Arqueología. ¿Cómo se puede lograr esto? La única forma de mantener proyectos nacionales de investigación es que la Dirección de Arqueología tenga acceso a un mayor presupuesto anual para lograr desarrollar las múltiples actividades que demanda y los proyectos de investigación. Adicionalmente, todo proyecto de investigación arqueológica extranjero debería contar con un codirector nacional, asegurando de esa manera espacios laborales para los colegas nacionales y el buen manejo del patrimonio cultural arqueológico del país.

En relación con la formación educativa, es necesario incentivar y apoyar a estudiantes graduados en la obtención de becas de posgrado en los niveles de maestrías y doctorados en Arqueología y Antropología en el exterior. También se podría diseñar e implantar un programa de posgrado a nivel de maestría en Arqueología y en Antropología en el país. En la medida que este cuente con más arqueólogos con maestrías y doctorados, la arqueología salvadoreña se podrá consolidar, creando una masa crítica que tanto hace falta hoy en día.

Por otro lado, mucha de la responsabilidad por la carencia de una arqueología salvadoreña recae en todos los arqueólogos y arqueólogas nacionales, debido a que han tenido que transcurrir dos décadas para finalmente poder conformar una asociación de arqueología salvadoreña. A principios del presente año se inició el proceso de inscripción de la Asociación de Arqueología de El Salvador (Arques) ante el Ministerio de Gobernación. Se espera que para finales del 2015 esta asociación esté debidamente registrada y trabajando en pro de la arqueología salvadoreña.

Medio siglo ha transcurrido desde que el arqueólogo norteamericano Stanley Boggs asumió la jefatura del Departamento de Excavaciones Arqueológicas en nuestro país. Muchas cosas han cambiado en la arqueología de El Salvador desde aquel tiempo; muchas de ellas positivas. Sin embargo, algunos aspectos no han evolucionado aún, como la conformación de una arqueología salvadoreña y el hecho de que el manejo del patrimonio cultural arqueológico todavía este bajo la dirección de un especialista extranjero, cambiando solamente el país de su origen. 


\section{Referencias}

Amaroli, Paul (1984). "Cara Sucia: nueva luz sobre el pasado de la Costa Occidental de El Salvador". Universitas 1(1):15-19. San Salvador.

Andrews, E.; Wyllys, V. (1986). The Archaeology of Quelepa, El Salvador. Middle American Research Institute, Pub. 42. Tulane University, New Orleans.

Boggs, Stanley H. (1944). "Excavations in Central and Western El Salvador. Appendix C to Archaeological Investigations in El Salvador. Memoirs of the Peabody Museum of Archaeology and Ethnology", Harvard University 9(2). Cambridge. (1962) Excavations at Tazumal, El Salvador. Year Book of the American Philosophical Society, pp. 488-491.

(1963) Excavations at Tazumal, El Salvador. Year Book of the American Philosophical Society, pp. 505-507.

Bruhns, Karen Olsen (1980). "Cihuatan: An Early Postclassic town of El Salvador: The 1977-1978 Excavations". University of Missouri Monographs in Anthropology No. 5. Department of Anthropology, University of Missouri, Columbia.

Castellón, Blas Román (1992). “Bibliografía de Arqueología de El Salvador". En Cuadernos de Arquitectura Mesoamericana. No. 22. Seminario de Arquitectura Prehispánica, Centro de Investigaciones en Arquitectura, Unam, México.

Cobos, Rafael (1994). Síntesis de la Arqueología de El Salvador (1850-1991). Colección Antropología e Historia No. 21. Consejo Nacional para la Cultura y el Arte, Dirección General de Publicaciones e Impresos.

Demarest, Arthur (1986). The Archaeology of Santa Leticia and the Rise of Maya Civilization. Middle American Research Institute, Pub. 52. Tulane University, New Orleans.

Erquicia, Heriberto (2011). "A quince años de la formación de arqueólogos en El Salvador, 1995-2010". Memorias del XXV Simposio de Investigaciones Arqueologicas en Guatemala.

Escamilla, Marlon y William Fowler (2011). "Práctica y conducta de la arqueología salvadoreña durante los últimos 25 años: el inicio de una arqueología nacional". Memorias del XXV Simposio de Investigaciones Arqueologicas en Guatemala.

Fowler, William Jr. (1981). "The Pipil-Nicarao of Central America". Tesis Doctoral, University of Calgary. (1989) The Cultural Evolution of Ancient Nahua Civilizations: the Pipil-Nicarao of Central America. University of Oklahoma Press, Norman.
Fowler, William y Howard Earnest (1985). Settlement Patterns and Prehistory of the Paraíso Basin of El Salvador. Journal of Field Archaeology 12:19-32.

Fowler Jr., William R., Francisco Estrada-Belli, Jennifer R. Bales, Matthew D. Reynolds y Kenneth L. Kvamme (2007). "Landscape Archaeology and Remote Sensing of a Spanish-Conquest Town: Ciudad Vieja, El Salvador". En Remote Sensing in Archaeology. Interdisciplinary Contributions to Archaeology, (editado por J. Wiseman y Farouk El-Baz). pp. 395-421. Springer, New York.

Fowler, William R. (Editor) (2011). Ciudad Vieja: Excavaciones, arquitectura y paisaje cultural de la primera villa de San Salvador. Editorial Universitaria, Secretaría de Cultura de la Presidencia, San Salvador.

Haberland, Wolfgang (1958). "Ceramic Sequences in El Salvador". American Antiquity 26:21-29.

Longyear III, John M., (1944). "Archaeological Investigations in El Salvador". Memoirs of the Peabody Museum of Archaeology and Ethnology Vol. 9, No. 2. Harvard University, Cambridge.

Ohi, Kuniaki (2000). Chalchuapa, Memoria Final de las Investigaciones Interdisciplinarias de El Salvador. Universidad de Estudios Extranjeros de Kyoto, Kyoto, Japón.

Paredes, Federico y Erquicia, Heriberto (2013). “Los conceptos de pasado histórico, Estado y patrimonio como elementos indispensables para la elaboración de una biografía crítica de la arqueología salvadoreña". Identidades 4 (6).

Sharer, Robert (1978). The Prehistory of Chalchuapa, EI Salvador. 3 vol. University of Pennsylvania Press, Philadelphia.

Sheets, Payson (2006). The Ceren Site: An ancient village buried by volcanic ash in Central America. Thomson Higher Education, Belmont, CA.

Sheets, Payson (Editor) (1983). Archaeology and Volcanism in Central America: The Zapotitán Valley of El Salvador. University of Texas Press, Austin.

Valdivieso, Fabricio (2010). "Remembranzas de un departamento de arqueología con los primeros arqueólogos formados en El Salvador". Revista de museología Kóot 1 (2), del Museo Universitario de Antropología, MUA, de la Universidad Tecnológica de El Salvador. 